\title{
Imaging the Microworld
}

In the first century A.D., the Roman philosopher Seneca wrote that "letters, however small and indistinct, are seen enlarged and more clearly through a globe of glass filled with water." This was the first written record of anyone using a tool to enlarge the image of an object. Since then, various types of microscopes have shown us worlds on an entirely different scalefrom the fine structures of materials to living cells and to individual atoms.

The first true light microscopes appeared in the early 17 th century, when inventors could create practical lens designs. In 1590 Zacharias Janssen in the Netherlands proposed a compound microscope using two tiny lenses as an objective (a convex lens) and an eyepiece (a concave lens). The convex-concave combination yielded an erect image for the viewer, but required a very long tube to hold both lenses at the appropriate distance from each other. By 1612, physicist and astronomer Galileo Galilei had designed and made several compound microscopes of his own.

Many later microscope designs used a convex-convex combination that yielded an inverted image. This idea was proposed as a telescope by German astronomer Johannes Kepler in 1611, but the first such microscope was not constructed until 1628 by Christoph Scheiner, who used it for visual observation and microprojection.

The most well-known microscope studies in the 1600 s were made with singlelens, or simple, microscopes. In Holland, Anton van Leeuwenhoek, a superior craftsman, made his own instruments. In 1671 he became interested in the small lenses used by drapers to inspect the quality of their cloth. He ground his own minute lenses from globules of glass clamped between two small perforated metal plates.

Of the estimated 500 to 600 microscopes Leeuwenhoek made, only nine still exist. The best surviving one achieves $270 \mathrm{X}$ magnification and resolution to $1.4 \mu \mathrm{m}$, although Leeuwenhoek's records indicate that he created lenses capable of 500X magnification with resolution to $1 \mu \mathrm{m}$.

In the same period, Robert Hooke of England made contributions that are fundamental to current microscopes. He introduced illumination systems and holding stages and coarse and fine adjustment knobs for more accurate focusing.

Because the optics of these early microscopes were uncorrected, all instruments suffered from chromatic and spherical aberration. Achromatic lenses were devel- oped in the middle of the 18th century and used in telescope optics, but the small size of microscope lenses precluded such a switch. In the mid-19th century, Charles A. Spencer introduced higher numerical apertures in microscope objectives, and he also improved color correction by using glass-fluorite combinations for his lenses. Later that century, Ernst Abbe designed an apochromatic objective to be used with a set of compensating eyepieces.

By far the most extraordinary advance in instruments for microscopy resulted from Louis de Broglie's hypothesis in 1924 that all material particles, including electrons, possess a wave nature. In 1926 Erwin Schrödinger began to publish his work on wave mechanics; in the same year Hans Busch in Germany realized the practical significance of this work. Since electrons have wavelengths orders of magnitude shorter than visible light, they can potentially resolve smaller objects. Busch, who had been studying the trajectories of electrons in magnetic fields for about 15 years, used Schrodinger's theory to propose that axially symmetric magnetic or electric fields could act as lenses for electrons or other charged particles. In 1927 Busch created a magnetic lens to verify his theory. Four years later C.J Davisson and C.J. Calbick in the United States created a corresponding lens with an electric field.

The following year in Germany, Ernst Brüche and $\mathrm{H}$. Johannson used electrostatic lenses and a 300-eV electron beam to produce images of a heated oxide cathode. Also in 1932 in Germany, M. Knoll and Ernst Ruska used magnetic lenses and 60$\mathrm{keV}$ electrons to produce images of the electron source in a discharge tube. Knoll and Ruska first claimed that an electron microscope using high-velocity electrons could surpass the resolution of a light microscope by a large margin. By 1936 emission-type electron microscopes for observing the fine structure of electronemitting cathode materials had become quite sophisticated. The maximum resolution of these microscopes was about $400 \AA$

Many materials, however, do not readily emit electrons. In $1931 \mathrm{~K}$. Rudenberg filed for a German patent on a transmission electron microscope that could be used for studying many other substances. By 1934 Knoll and Ruska had described their construction of an electron microscope that could attain high resolution with transmitted electrons, using magnetic fields as lenses. Also at this time, L. Marton in Bel- gium first attempted to use an electron microscope to study biological materials.

Finally, in 1935 E. Driest and H.O. Müller exceeded the resolution of the light microscope by modifying one of Ruska's instruments, using suggestions made by Ruska himself. F. Krause continued the work on Ruska's modified instrument to obtain electron micrographs of various materials. Krause showed that materials would not be destroyed by the impinging electron beam if the specimens were sliced thinly enough. (An earlier theory proposed that specimens had to be "stained" to retain outlines of their forms after the electron beam disintegrated them.)

Working with $\mathrm{B}$. von Borries for the firm of Siemens and Halske, Ruska developed an improved electron microscope for general laboratory use in 1938. At the same time, A. Prebus and J. Hillier in Toronto designed another practical magnetic electron microscope. Both instruments could resolve details down to $100 \AA$.

Refinements came rapidly. In 1939 Hans Mahl in Germany invented an electrostatic electron microscope capable of high resolution. Also that year, L. Marton (who had used electron microscopes to study biological materials) designed a magnetic electron microscope for RCA, using electronically regulated power supplies. A year later, RCA was the first company to offer a commercially available electron microscope; the RCA instrument was capable of $20 \AA$ resolution. Meanwhile, Ruska and von Borries had improved the resolution of the Siemens instrument to $22 \AA$. In 1945 Hillier, still working in Toronto, described a way to make the magnetic objective accurately symmetrical, which would improve the resolution to $10 \AA$. Refinements have brought this down to $5 \AA$.

Major breakthroughs continued, including the development of scanning electron microscopy, first proposed by Young and Roberts in 1951. In the SEM, a set of scanning coils deflects the electron beam at various rates across a material specimen, building up a complete image. This technique yields striking images of threedimensional surfaces caused by the secondary electrons ejected from the sample's surface.

Though optical and electron microscopy remain the workhorses, many other specialized imaging techniques have been developed to study materials-field ion microscopy, scanning tunneling microscopy, and atomic force microscopy. Instruments have reached the quantummechanical limits of observability, but new approaches continue to improve our observations of the microworld.

Kevin J. Anderson 


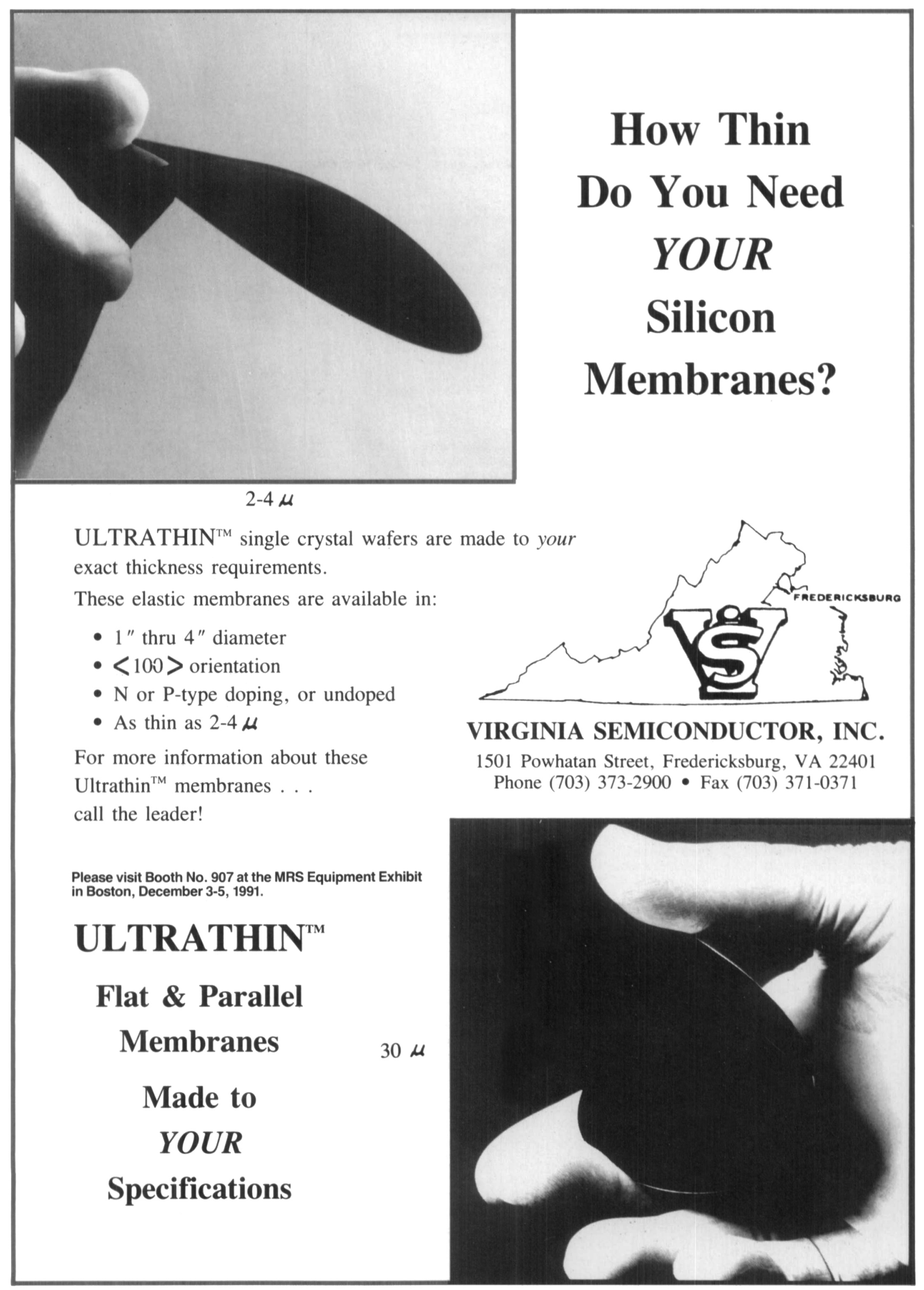

